\title{
Spin alignment measurements of vector mesons with ALICE at the LHC
}

\author{
Sourav Kundu (for the ALICE Collaboration)* \\ School of Physical Sciences, National Institute of Science \\ Education and Research, HBNI, Jatni 752050, India
}

\section{Introduction}

In non-central heavy-ion collisions a large initial angular momentum is expected to be created. A theoretical study predicts the magnitude of the angular momentum to be $\sim 10^{5} \hbar[1]$. In presence of large initial angular momentum, vector mesons $($ spin $=1)$ can be polarized due to the spin-orbital interaction of quantum chromodynamics. Polarization/spin alignment measurements of vector meson provide a unique opportunity to probe this initial condition in heavy-ion collisions. Spin alignments can be studied by measuring the angular distributions of the decay daughters of vector mesons with respect to the quantization axis. The quantization axis can be perpendicular to the production plane, defined by the momentum of the vector meson and the beam axis or it can be perpendicular to the reaction plane, subtended by the impact parameter and the beam axis. In the experiment the event plane is used as a proxy of the reaction plane (as the impact parameter direction is not directly measured) and the results are further corrected for the event plane resolution. The angular distribution is expressed as [2],

$$
\frac{\mathrm{d} N}{\mathrm{~d} \cos \theta^{*}} \propto\left[1-\rho_{00}+\cos ^{2} \theta^{*}\left(3 \rho_{00}-1\right)\right] .
$$

where $\theta^{*}$ is the angle made by the decay daughter of vector meson with the quantization axis in the rest frame of the vector meson. $N_{0}$ is the normalization constant. $\rho_{00}$ is the second diagonal element of the $3 \times 3$ hermitian spin density matrix. $\rho_{00}$ corresponds to the probability of finding a vector meson in

*Electronic address: sourav.kundu@cern. ch spin state 0 , where vector mesons can occupy 3 spin states $-1,0$ and 1 . In absence of spin alignment all spin states are equally probable which makes $\rho_{00}=1 / 3$ and leads to a flat angular distribution. Deviation of $\rho_{00}$ from $1 / 3$ would lead to a non-uniform angular distribution which is the experimental evidence of the presence of large initial angular momentum, followed by the spin alignment of vector mesons.

We present the recent ALICE measurements of transverse momentum $\left(p_{\mathrm{T}}\right)$ and centrality dependence of $\rho_{00}$ for $\mathrm{K}^{* 0}$ and $\phi$ vector mesons in $\mathrm{Pb}-\mathrm{Pb}$ collisions at $\sqrt{s_{\mathrm{NN}}}=2.76 \mathrm{TeV}$. Measurements are also compared with the results from $\mathrm{pp}$ collisions where we do not expect the presence of initial angular momentum and with the spin zero hadron $\mathrm{K}_{\mathrm{S}}^{0}$ in $\mathrm{Pb}-\mathrm{Pb}$ collisions as a null test.

\section{Analysis details}

This analysis is carried out by analyzing 14 million and 43 million minimum bias $\mathrm{Pb}-\mathrm{Pb}$ collisions at $\sqrt{s_{\mathrm{NN}}}=2.76 \mathrm{TeV}$ and pp collisions at $\sqrt{s}=13 \mathrm{TeV}$, respectively. The measurements are performed at midrapidity $(|y|<0.5)$. Details about the ALICE detector, event selection, trigger logic, track reconstruction, centrality determination and $2^{\text {nd }}$ order event plane estimation using the V0 detectors are discussed in $[3,4]$. The $\mathrm{K}^{* 0}$ and $\phi$ vector mesons are reconstructed from the invariant mass distribution of oppositely charged $\mathrm{K} \pi$ and KK pairs, respectively as discussed in $[4,5]$. Whereas, the $\mathrm{K}_{\mathrm{S}}^{0}$ are reconstructed from the invariant mass distribution of oppositely charged $\pi \pi$ pairs, where the $\pi \pi$ pairs are selected by using V0 decay topology [6]. Charged $\pi$ and $\mathrm{K}$ mesons are identified by using two particle identification techniques: the specific energy loss measured in the Time Pro- 
jection Chamber (TPC) and the velocity measured by the Time-Of-Flight (TOF) detector. The $\mathrm{K}^{* 0}, \phi$ and $\mathrm{K}_{S}^{0}$ signals are extracted for various $p_{\mathrm{T}}$ and $\cos \theta^{*}$ bins in different centrality classes and corrected for detector acceptance $\times$ efficiency to get the corrected yields. The efficiency and acceptance corrected $\cos \theta^{*}$ distributions [5] are fitted with Eq.1 to extract $\rho_{00}$ values for each studied $p_{\mathrm{T}}$ interval in the different centrality classes.

\section{Results}

The extracted $\rho_{00}$ values for $\mathrm{K}^{* 0}$ as a function of $p_{\mathrm{T}}$ in $10-50 \% \mathrm{~Pb}-\mathrm{Pb}$ collisions at $\sqrt{s_{\mathrm{NN}}}$ $=2.76 \mathrm{TeV}$ are shown in Fig. 1. The results are compared with the measurements from pp collisions at $\sqrt{s}=13 \mathrm{TeV}$ and with $\mathrm{K}_{\mathrm{S}}^{0}$ measurements in $20-40 \% \mathrm{~Pb}-\mathrm{Pb}$ collisions at $\sqrt{s_{\mathrm{NN}}}=2.76 \mathrm{TeV}$. The extracted $\rho_{00}$ values for $\mathrm{K}^{* 0}$ in $\mathrm{pp}$ collisions and for $\mathrm{K}_{\mathrm{S}}^{0}$ in $\mathrm{Pb}-\mathrm{Pb}$ collisions are consistent with $1 / 3$ through out the whole measured $p_{\mathrm{T}}$ intervals. Whereas, the $\rho_{00}$ values for $\mathrm{K}^{* 0}$ in $\mathrm{Pb}-\mathrm{Pb}$ collisions show a deviation from $1 / 3$ at $p_{\mathrm{T}}<2 \mathrm{GeV} / c$. Fig-

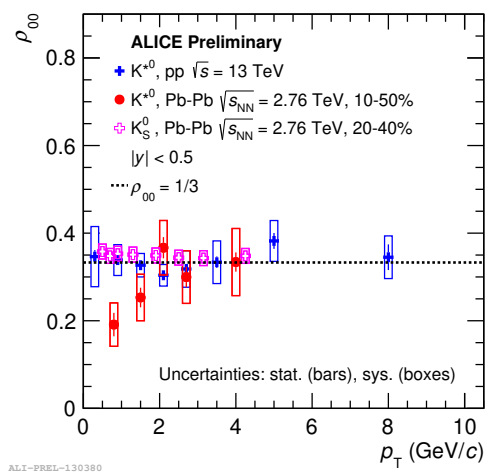

FIG. 1: (Color online) $\rho_{00}$ vs. $p_{\mathrm{T}}$ for $\mathrm{K}^{* 0}$ in 10 $50 \% \mathrm{~Pb}-\mathrm{Pb}$ collisions at $\sqrt{s_{\mathrm{NN}}}=2.76 \mathrm{TeV}$, along with the results for $\mathrm{K}^{* 0}$ in pp collisions at $\sqrt{s}=$ $13 \mathrm{TeV}$ and for $\mathrm{K}_{\mathrm{S}}^{0}$ in $20-40 \% \mathrm{~Pb}-\mathrm{Pb}$ collisions at $\sqrt{s_{\mathrm{NN}}}=2.76 \mathrm{TeV}$.

ure 2 shows $\rho_{00}$ vs. $\left\langle\mathrm{N}_{\text {part }}\right\rangle$ for $\mathrm{K}^{* 0}$ and $\phi$ mesons in $\mathrm{Pb}-\mathrm{Pb}$ collisions at $\sqrt{s_{\mathrm{NN}}}=2.76$ $\mathrm{TeV}$. The $\rho_{00}$ values for vector mesons in $\mathrm{Pb}-$ $\mathrm{Pb}$ collisions show a clear centrality dependence. Maximum deviation from 1/3 occurs at mid-central collisions. In peripheral and central collisions the measurements are consistent with $1 / 3$ within uncertainty. The centrality dependence of the extracted $\rho_{00}$ values are similar to the centrality dependence of the angular momentum [1].

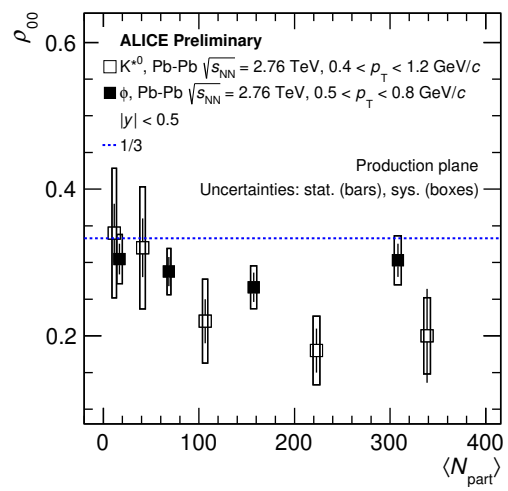

FIG. 2: (Color online) $\rho_{00}$ vs. $\left\langle\mathrm{N}_{\text {part }}\right\rangle$ for $\mathrm{K}^{* 0}$ and $\phi$ mesons in $10-50 \% \mathrm{~Pb}-\mathrm{Pb}$ collisions at $\sqrt{s_{\mathrm{NN}}}=$ $2.76 \mathrm{TeV}$.

\section{Summary}

A first observation of the spin alignment of vector mesons in high energy heavy-ion collisions is presented. The extracted $\rho_{00}$ values for vector mesons in heavy-ion collisions deviate from $1 / 3$ at $p_{\mathrm{T}}<2 \mathrm{GeV} / c$. The deviation from $1 / 3$ is largest at mid-central collisions which is expected from the presence of a large initial angular momentum at mid-central collisions.

\section{References}

[1] F. Becattini, F. Piccinini and J. Rizzo, Phys. Rev. C 77, 024906 (2008).

[2] K. Schilling, P. Seyboth and G. E. Wolf, Nucl. Phys. B 15, 397 (1970).

[3] B. Abelev et al. [ALICE Collaboration], Int. J. Mod. Phys. A 29, 1430044 (2014).

[4] J. Adam et al. [ALICE Collaboration], Phys. Rev. C 95, 064606 (2017).

[5] B. Mohanty [ALICE Collaboration], EPJ. Web. Conf. 171, 16008 (2018).

[6] B. Abelev et al. [ALICE Collaboration], Phys. Rev. Lett. 111, 222301 (2013). 